\title{
Association of miR-196a2, miR-27a, and miR- 499 polymorphisms with isolated congenital heart disease in a Chinese population
}

\author{
K. Yu ${ }^{1,2}$, Y. Ji ${ }^{1}$, H. Wang ${ }^{1}$, Q.K. Xuan' ${ }^{1}$, B.B. Li ${ }^{1}$, J.J. Xiao ${ }^{3}$, W. Sun ${ }^{1}$ and \\ X.Q. Kong ${ }^{1}$ \\ ${ }^{1}$ Department of Cardiology, \\ The First Affiliated Hospital of Nanjing Medical University, Nanjing, \\ Jiangsu Province, China \\ ${ }^{2}$ Department of Cardiology, Huai'an First People's Hospital, \\ Nanjing Medical University, Huai'an, Jiangsu Province, China \\ ${ }^{3}$ Regeneration and Ageing Lab, Experimental Center of Life Sciences, \\ School of Life Science, Shanghai University, Shanghai, China; \\ Shanghai Key Laboratory of Bio-Energy Crops, School of Life Sciences, \\ Shanghai University, Shanghai, China \\ Corresponding authors: X.Q. Kong / W. Sun \\ E-mail: huaianyk@163.com
}

Genet. Mol. Res. 15 (4): gmr15048929

Received June 29, 2016

Accepted August 8, 2016

Published October 17, 2016

DOI http://dx.doi.org/10.4238/gmr15048929

Copyright (C) 2016 The Authors. This is an open-access article distributed under the terms of the Creative Commons Attribution ShareAlike (CC BY-SA) 4.0 License.

\begin{abstract}
We hypothesized that single nucleotide polymorphisms (SNPs) in certain microRNAs contribute to congenital heart disease (CHD) phenotypes. Five hundred and seventy-three subjects were enrolled in this study. DNA extracted from peripheral blood cells was used for SNP genotyping of miR-196a2 (rs11614913), miR27a (rs11671784, rs895819), and miR-499 (rs3746444). Allele and genotype association analyses were performed to evaluate the
\end{abstract}

Genetics and Molecular Research 15 (4): gmr15048929 
correlation between certain microRNA SNPs and three phenotypes of isolated CHD: atrial septal defect (ASD), ventricular septal defect (VSD), and patent ductus arteriosus (PDA). All the participants carried a homozygous CC variant of miR-27a (rs11671784). The homozygous $\mathrm{CC}$ variant of miR-196a2 (rs11614913, T $>C$ ) was negatively associated with ASD compared with the wild-type TT variant $(\mathrm{OR}=0.379,95 \% \mathrm{CI}$ $=0.209-0.686, \mathrm{P}=0.001)$. The miR-196a2 $\mathrm{C}$ allele was negatively associated with ASD compared with the T allele $(\mathrm{OR}=0.646,95 \% \mathrm{CI}$ $=0.491-0.849, \mathrm{P}=0.002)$. The statistically significant results were further confirmed by dominant and recessive model assays. SNPs of miR-27a (rs895819, T>C) and miR-499 (rs3746444, A $>$ G) showed diverse association with ASD, VSD, or PDA, but the differences were not statistically significant. The rs11614913 (T>C) SNP of miR-196a2 is associated with ASD, and the homozygous $\mathrm{CC}$ variant and the $\mathrm{C}$ allele are protective factors associated with ASD. The homozygous CC variant and the $\mathrm{C}$ allele of the rs11614913 (T>C) SNP of miR-196a2 are associated with a significantly reduced risk of ASD.

Key words: Single nucleotide polymorphisms; Congenital heart disease; microRNA; miR-196a2

\section{INTRODUCTION}

Congenital heart disease (CHD), defined as clinically structural heart defects present before and at birth, is the leading cause of serious infant morbidity and mortality worldwide. The incidence of CHD at birth was 10-12 per 1000 live births in 2013 (Hoffman, 2013). Similarly, in China the overall incidence based on epidemiological studies was 8 per 1000 live births in 2011, an approximate increase of 6.7 times compared with 1996 (Yu et al., 2014).

CHD can be classified into three broad categories in accordance with clinical manifestations: cyanotic heart disease, left-sided obstruction defects, and septation defects (Bruneau, 2008). The condition includes transposition of the great arteries (TGA), tetralogy of Fallot (TOF), tricuspid atresia (TA), pulmonary atresia (PA), Ebstein's anomaly of the tricuspid valve, double outlet right ventricle (DORV), persistent truncus arteriosus (PTA), total anomalous pulmonary venous connection (TAPVC), hypoplastic left heart syndrome (HLHS), mitral stenosis (MS), aortic stenosis (AS), aortic coarctation (AC), interrupted aortic arch (IAA), atrial septal defect (ASD), ventricular septal defect (VSD), atrioventricular septal defect (AVSD), bicuspid aortic valve (BAV), patent ductus arteriosus (PDA), and pulmonary stenosis (PS). Approximately $80 \%$ of patients have heart defects without underlying systemic disease (isolated CHD or non-syndromic CHD). In China, CHD has been clinically categorized by lesion location in a traditional way, and most patients who survive to adulthood are diagnosed with ASD, VSD, PDA, TOF, PS, etc., which constitute the most common classifications of isolated CHD. Pain and illness place an enormous burden on patients and their families; this is especially true in China owing to delayed childbearing and the one-child policy. Therefore, solutions that allow early prevention and diagnoses are urgently required.

The pathogenesis of CHD remains unclear. Until recently, the major underlying defects were thought to be mutations in the regulators of heart development (Srivastava, 2006). That

Genetics and Molecular Research 15 (4): gmr15048929 
is to say, perturbations in protein-mediated transcriptional control and signaling systems were thought to contribute to heart defects at the transcriptional level (Schonrock et al., 2012). However, a brand-new research field has emerged over the past two decades that emphasizes post-transcriptional factors. Genomic sequencing has shown that protein-coding regions account for just $1.5 \%$ of the human DNA genome (Lander et al., 2001); the remaining DNA is involved in the production of noncoding RNAs, which constitute 96\% of cells (Patrushev and Kovalenko, 2014). Studies indicate that thousands (30-50\%) of human genes are the targets of noncoding RNAs called microRNAs (miRNAs, miRs) (Lewis et al., 2005). miRNAs are evolutionarily conserved, single-stranded, noncoding, small (20-26 nucleotides in length) RNAs that negatively regulate gene expression by affecting mRNA stability and/or translation (Bartel, 2009). Such small noncoding RNAs act at the post-transcriptional level as "fine tuners" and/or "safeguards" to balance dramatic environmentally induced alterations in gene expression and maintain the homeostasis of organisms. Certain miRNAs are critical to heart development and are tissue-specific. Even slight perturbations in programming during cardiogenesis, which are mostly, if not exclusively, caused by single nucleotide polymorphisms (SNPs), may alter miRNA expression and/or maturation. This can cause incompatibility between the microRNAs and their target mRNAs, which contributes to abnormal function and transcription of the target genes, disturbing the regulation and translation of proteins (Chen and Wang, 2012). However, only $10 \%$ of human pre-miRNAs and less than $1 \%$ of miRNAs have been detected in seed regions (Saunders et al., 2007). Recent studies have shown that miR-196a2 might play a role in the subtle temporal and spatial regulation processes that occur during heart development through the miR-196a-HOXB8-Sonic hedgehog signaling pathway (Xu et al., 2009; Li et al., 2016). miR-499 and miR-27a disturb differentiation and proliferation in cardiomyocyte development by regulating the expression of myosin heavy chain beta (MHC- $\beta$ ) and SOX-6 (Nishi et al., 2011; Wang et al., 2013). Hence, we hypothesized that SNPs of miRNAs might contribute to dysregulation of heart development and lead to CHD phenotypes. We selected the four SNPs located in the pre-miRNAs mentioned previously (rs11614913 in miR-196a2, rs895819 and rs11671784 in miR-27a, and rs3746444 in miR-499) to test the hypothesis by genotyping analysis in a case-control study (CHD group and control group). We hope that this study will improve genotype screening in CHD.

\section{MATERIAL AND METHODS}

\section{Patients}

This study was approved by the institutional review board of Nanjing Medical University, Nanjing, China, and was conducted in accordance with the ethical standards formulated in the Helsinki Declaration. Subjects in this case-control study were all genetically unrelated ethnic Han Chinese, and were recruited from the First Affiliated Hospital of Nanjing Medical University between May 2011 and September 2013. Isolated CHD without underlying systemic disease was diagnosed by Doppler echocardiography and confirmed by interventional and/or surgical operation; no genetic kinship was involved. Those who had a family history of CHD in a first-degree relative were excluded. Exclusion criteria also included phenylketonuria, maternal diabetes mellitus, maternal teratogen exposures (e.g., biological agents, chemical agents, radioactive agents, and pesticides), maternal therapeutic drug exposures or viral infection, and allergies before and during pregnancy.

Genetics and Molecular Research 15 (4): gmr15048929 
K. Yu et al.

Those who had been through a life-threatening abortion and pharmaceutical therapy were also ruled out. The control subjects were healthy volunteers from the same geographic area as the cases, and were recruited at the same time; they were frequency-matched to the cases in terms of age ( \pm 5 years) and gender.

Structured questionnaires including baseline information were completed by trained interviewers after direct contact with subjects and collection of auxiliary examination and operation data. Venous blood (approximately $3 \mathrm{~mL}$ ) was collected in a resting state from each subject after overnight fasting.

\section{DNA extraction}

Blood samples were collected in ethylenediaminetetraacetic acid-containing tubes. Genomic DNA was extracted from whole blood using the Qiagen Blood Midi Kit (Spin Protocol, Qiagen, Duesseldorf, Germany). The purity and concentration of DNA were determined using a NanoDrop $2000 \mathrm{UV} /$ Vis spectrophotometer. The $\mathrm{OD}_{260} / \mathrm{OD}_{280}$ [the ratio of optical density (OD) at $260 \mathrm{~nm}$ to that at $280 \mathrm{~nm}$ ] ratios for all samples were approximately 1.8 .

\section{SNP selection and genotyping assays}

We selected three miRNAs that were doubtful or had been identified as closely related to heart development by retrieving the relevant literature: miR-196a2 (Xu et al., 2009), miR27a (Nishi et al., 2011), and miR-499 (Sluijter et al., 2010). We then checked a certain number of SNP loci in the National Center for Biotechnology Information (NCBI) database (http:// www.ncbi.nlm.nih.gov) and the HapMap database (http://www.hapmap.org). The reported minor allele frequency (MAF) was $\geq 0.05$. Of all the SNPs, rs11614913 of miR-196a2 (MAF $=0.449)$ and $\mathrm{rs} 895819$ of $\mathrm{miR}-27 \mathrm{a}(\mathrm{MAF}=0.290)$ met the requirement for high quality experiments. No frequency data for miR-27a (rs11671784) or miR-499 (rs3746444) were retrieved, but they deserve further exploration.

Genotyping was carried out using Taqman ${ }^{\circledR}$ SNP genotyping assays. The sequences of the four miRNA SNPs were as follows: TTTTGAACTCGGCAACAAGAAACTG[C/T] CTGAGTTACATCAGTCGGTTTTCGT for rs11614913; CTTAGCCACTGTGAACACGAC TTGG[C/T]GTGGACCCTGCTCACAAGCAGCTAA for rs895819; ATGTTTAACTCCTC TCCACGTGAAC[G/A]TCACAGCAAGTCTGTGCTGCTTCCC for rs3746444; and TGTG AGCAGGGT[T/CICAC for rs11671784. The polymerase chain reaction (PCR) amplifications were run on a 7900HT Fast instrument (Applied Biosystems, Grand Island, NY, USA) on 384-well PCR plates; the reaction mixture ( $5 \mu \mathrm{L}$ total volume) comprised $1.25 \mu \mathrm{L}$ DNasefree water, $20 \mathrm{ng}$ genome DNA $(20 \mathrm{ng} / \mu \mathrm{L}), 2.5 \mu \mathrm{L} 2 \mathrm{X}$ Taqman ${ }^{\circledR}$ Universal PCR Master Mix without AmpErase ${ }^{\circledR} \mathrm{UNG}$, and $0.25 \mu \mathrm{L} 20 \mathrm{X}$ Taqman $^{\circledR}$ SNP genotyping assay.

The thermal cycling conditions of the PCR ( 40 cycles) were as follows: holding for 10 min at $95^{\circ} \mathrm{C}$; denaturing for $15 \mathrm{~s}$ at $92^{\circ} \mathrm{C}$; and annealing/extension for $1 \mathrm{~min}$ at $60^{\circ} \mathrm{C}$. The final data were analyzed using SNP Genotyper Software (Applied Biosystems). Two experimenters independently examined the genotype pictures and repeated the assays if they did not reach a consensus. Ten percent of the samples were randomly selected for repeated assays for each locus, and the results were $100 \%$ concordant.

Genetics and Molecular Research 15 (4): gmr15048929 


\section{Statistical analysis}

Distributions of categorical variables are summarized as frequencies and percentages. Continuous data are reported as means \pm standard deviation. The Student $t$-test and the chi-square test were used for continuous data and categorical data, respectively. Logistic regression analysis was used to assess the association between SNPs and CHD onset. The Hardy-Weinberg equilibrium was tested using a goodness-of-fit chi-square test to compare the observed genotype frequencies with the expected ones among the control subjects. Corrections for multiple comparisons were performed between different groups and SNPs, and P values were obtained using the Bonferroni correction. All statistical analyses were performed using the Statistical Package for Social Sciences software for Windows (version 20.0).

\section{RESULTS}

A total of 573 local ethnic Han Chinese subjects were enrolled in this case-control study. The CHD patients was sub-grouped into an ASD group $(\mathrm{N}=174)$, a VSD group $(\mathrm{N}=$ $63)$, a PDA group $(\mathrm{N}=60)$, and a control group $(\mathrm{N}=276)$. The age [ASD $(40.81 \pm 13.56)$, $\operatorname{VSD}(34.84 \pm 11.81), \operatorname{PDA}(34.75 \pm 12.36) v s$ controls $(38.37 \pm 13.61)]$ and gender distribution [ASD (25.59\%), VSD (39.68\%), PDA (25.00\%) vs controls (30.07\%)] were comparable between case and control groups (Table 1).

Table 1. Baseline characteristics of congenital heart disease patients and control subjects.

\begin{tabular}{l|c|c|c}
\hline Characteristics & Control & Disease & P value \\
\hline ASD & 276 & $474(25.59)$ & 0.272 \\
\hline Male [N (\%)] & $83(30.07)$ & $40.81 \pm 13.56$ & 0.064 \\
\hline Age, years (means \pm SD) & $38.37 \pm 13.61$ & 63 & $25(39.68)$ \\
\hline VSD & 276 & $34.84 \pm 11.81$ & 0.299 \\
\hline Male [N (\%)] & $38.37 \pm 13.61$ & 60 & 0.059 \\
\hline Age, year (means \pm SD) & 276 & $15(25.00)$ & 0.433 \\
\hline PDA & $83(30.07)$ & $34.75 \pm 12.36$ & 0.059 \\
\hline Male [N (\%)] & $38.37 \pm 13.61$ & & \\
\hline Age, year (means \pm SD) & &
\end{tabular}

$\mathrm{ASD}=$ atrial septal defect; VSD = ventricular septal defect; PDA = patent ductus arteriosus.

The genotyping assays confirmed that all the participants were carriers of the homozygous CC variant at the miR-27a (rs11671784) locus, indicating that miR-27a rs 11671784 was not a polymorphic variation in our study population; therefore, this SNP was excluded from further analysis. The genotype distributions of the other three SNPs (miR196a2 rs11614913, miR-27a rs895819, and miR-499 rs3746444) are shown in Table 2 (ASD group $v s$ control group), Table 3 (VSD group $v s$ control group), and Table 4 (PDA group $v s$ control group) (Figures 1, 2, and 3). The observed genotype frequencies for these three polymorphisms were all in agreement with the Hardy-Weinberg equilibrium in the cases and controls $(\mathrm{P}>0.05)$. As shown in Table 2, the miR-196a2 (rs11614913, $\mathrm{T}>\mathrm{C})$ homozygous CC variant was negatively associated with ASD compared with the wild-type TT variant $(\mathrm{OR}=$ $0.379,95 \% \mathrm{CI}=0.209-0.686, \mathrm{P}=0.001)$. The miR-196a2 $\mathrm{C}$ allele was negatively associated with ASD compared with the $\mathrm{T}$ allele $(\mathrm{OR}=0.646,95 \% \mathrm{CI}=0.491-0.849, \mathrm{P}=0.002)$. The results were further confirmed by the dominant model $(\mathrm{OR}=0.626,95 \% \mathrm{CI}=0.417-0.939, \mathrm{P}$ $=0.023)$ and the recessive model $(\mathrm{OR}=0.455,95 \% \mathrm{CI}=0.266-0.776, \mathrm{P}=0.003)$. The miR-

Genetics and Molecular Research 15 (4): gmr15048929 
196a2 heterozygote CT variant showed a negative correlation with ASD compared with the wild-type TT variant, but the difference was not statistically significant $(\mathrm{OR}=0.741,95 \% \mathrm{CI}=$ 0.484-1.136, $\mathrm{P}=0.168$ ) (Table 2). In contrast, the miR-196a2 homozygous $\mathrm{CC}$ variant and the $\mathrm{C}$ allele were positively correlated with $\mathrm{VSD}(\mathrm{OR}=1.172,95 \% \mathrm{CI}=0.543-2.529, \mathrm{P}=0.950$; $\mathrm{OR}$ $=1.129,95 \% \mathrm{CI}=0.584-2.185, \mathrm{P}=0.718)$ and $\mathrm{PDA}(\mathrm{OR}=1.660,95 \% \mathrm{CI}=0.738-3.735, \mathrm{P}=$ $0.218 ; \mathrm{OR}=1.414,95 \% \mathrm{CI}=0.686-2.915, \mathrm{P}=0.346)$, respectively, compared with the wild-type TT variant. Moreover, the miR-196a2 C allele was positively correlated with VSD $(\mathrm{OR}=1.016$, $95 \% \mathrm{CI}=0.690-1.497, \mathrm{P}=0.934)$ and $\mathrm{PDA}(\mathrm{OR}=1.280,95 \% \mathrm{CI}=0.861-1.902, \mathrm{P}=0.221)$ compared with the $\mathrm{T}$ allele, but the differences were not statistically significant (Tables 3 and 4 ).
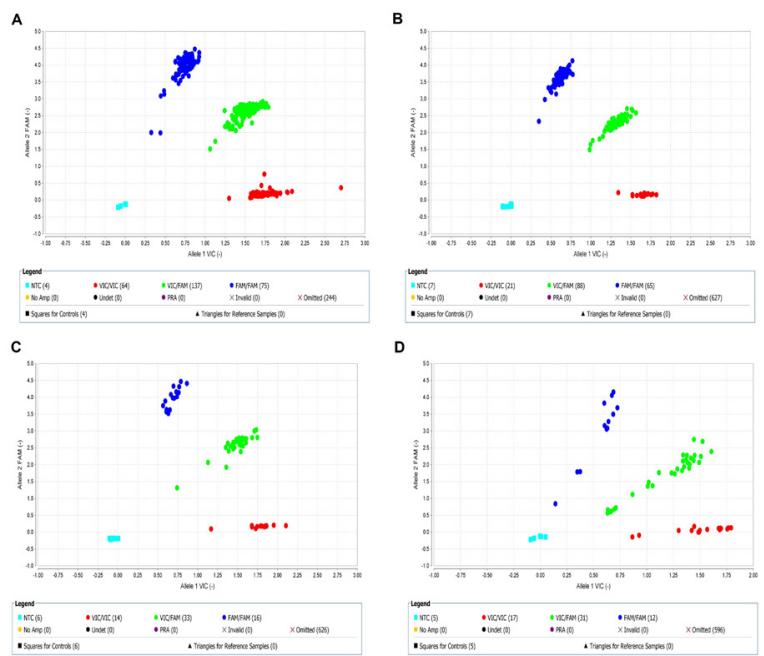

Figure 1. Genotype of rs11614913. A. control group; B. atrial septal defect (ASD) group; C. ventricular septal defect (VSD) group; D. patent ductus arteriosus (PDA) group.
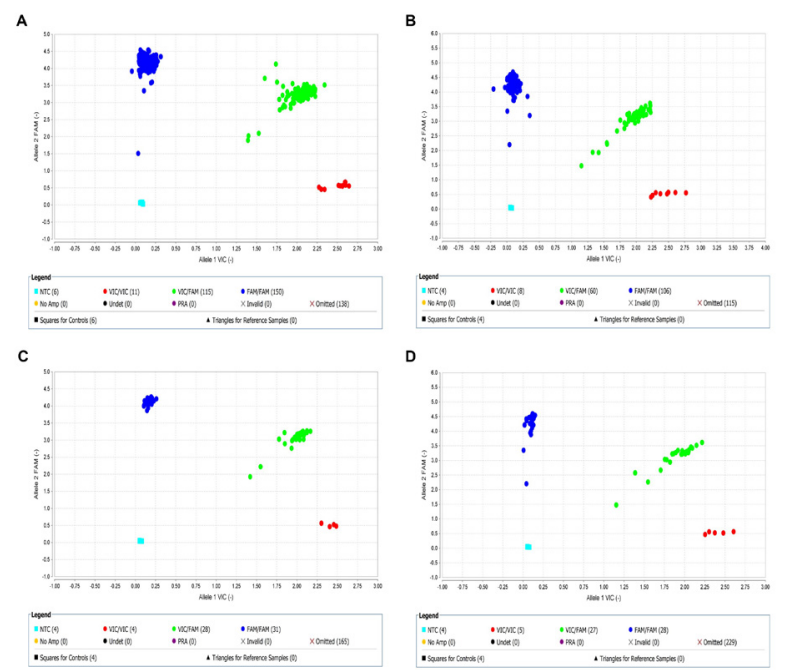

Figure 2. Genotype of rs895819. A. control group; B. atrial septal defect (ASD) group; C. ventricular septal defect (VSD) group; D. patent ductus arteriosus (PDA) group.

Genetics and Molecular Research 15 (4): gmr15048929 

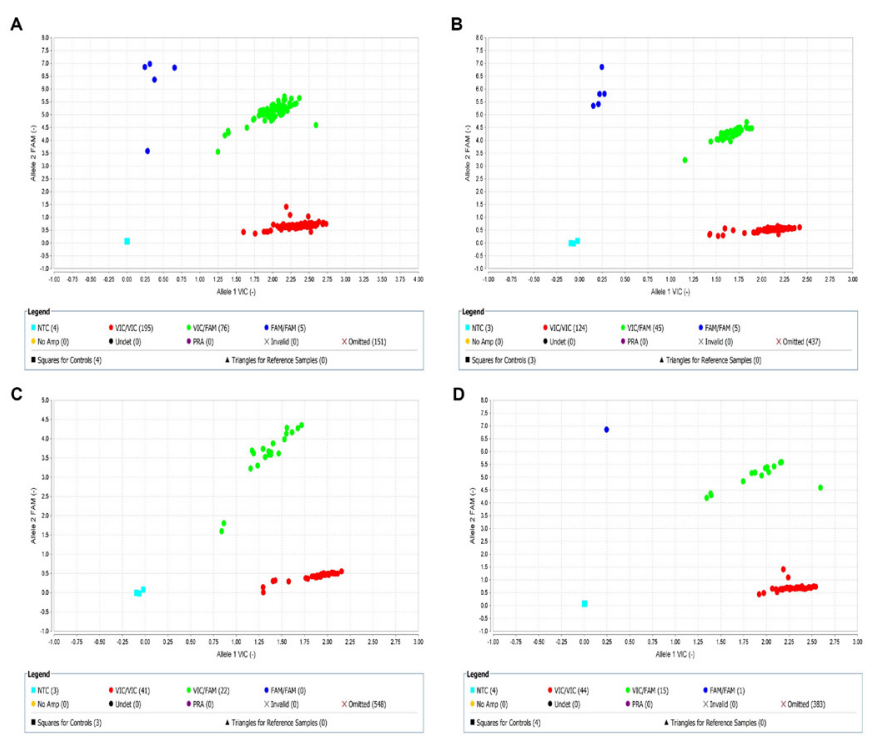

Figure 3. Genotype of rs3746444. A. control group; B. atrial septal defect (ASD) group; C. ventricular septal defect (VSD) group; D. patent ductus arteriosus (PDA) group.

Table 2. Differences in genotype frequencies of miRNA polymorphisms between atrial septal defect (ASD) patients and control subjects.

\begin{tabular}{|c|c|c|c|c|}
\hline Characteristics & Control $(\mathrm{N}=276)$ & $\operatorname{ASD}(\mathrm{N}=174)$ & OR $(95 \% \mathrm{CI})$ & P value \\
\hline \multicolumn{5}{|l|}{ miR-196a2 (rs11614913) } \\
\hline TT & 75 & 65 & 1.000 (reference) & \\
\hline $\mathrm{CT}$ & 137 & 88 & $0.741(0.484-1.136)$ & 0.168 \\
\hline $\mathrm{CC}$ & 64 & 21 & $0.379(0.209-0.686)$ & 0.001 \\
\hline Dominant model $(\mathrm{CC}+\mathrm{CT} v s \mathrm{TT})$ & 201 vs 75 & 109 vs 65 & $0.626(0.417-0.939)$ & 0.023 \\
\hline Recessive model (CC vs CT+TT) & $64 v s 212$ & $21 v s 153$ & $0.455(0.266-0.776)$ & 0.003 \\
\hline T allele & 287 & 218 & 1.000 (reference) & \\
\hline C allele & 265 & 130 & $0.646(0.491-0.849)$ & 0.002 \\
\hline HWE P & 0.925 & 0.288 & & \\
\hline \multicolumn{5}{|l|}{ miR-27a (rs895819) } \\
\hline TT & 150 & 106 & 1.000 (reference) & \\
\hline CT & 115 & 60 & $0.738(0.495-1.100)$ & 0.136 \\
\hline $\mathrm{CC}$ & 11 & 8 & $1.029(0.400-2.645)$ & 0.952 \\
\hline Dominant model $(\mathrm{CC}+\mathrm{CT} v s \mathrm{TT})$ & $126 v s 150$ & $68 v s 106$ & $0.764(0.519-1.123)$ & 0.170 \\
\hline Recessive model (CC vs CT+TT) & $11 v s 265$ & 8 vs 166 & $1.161(0.458-2.946)$ & 0.753 \\
\hline $\mathrm{T}$ allele & 415 & 272 & 1.000 (reference) & \\
\hline $\mathrm{C}$ allele & 137 & 76 & $0.846(0.615-1.165)$ & 0.306 \\
\hline HWE P & 0.053 & 0.894 & & \\
\hline \multicolumn{5}{|l|}{ miR-499 (rs3746444) } \\
\hline AA & 195 & 124 & 1.000 (reference) & \\
\hline GA & 76 & 45 & $0.931(0.605-1.434)$ & 0.746 \\
\hline GG & 5 & 5 & $1.573(0.446-5.543)$ & 0.703 \\
\hline Dominant model (GA+GG vs AA) & 81 vs 195 & $50 v s 124$ & $0.971(0.639-1.475)$ & 0.889 \\
\hline Recessive model (GG vs GA+AA) & 5 vs 271 & 5 vs 169 & $1.604(0.457-5.622)$ & 0.677 \\
\hline A allele & 466 & 293 & 1.000 (reference) & \\
\hline G allele & 86 & 55 & $1.017(0.704-1.470)$ & 0.928 \\
\hline HWE P & 0.437 & 0.710 & & \\
\hline
\end{tabular}

miRNA $=$ microRNA; OR $=$ odds ratio $; 95 \% \mathrm{CI}=95 \%$ confidence interval; HWE $=$ Hardy-Weinberg equilibrium. $\mathrm{P}$ values were adjusted using the Bonferroni correction.

Genetics and Molecular Research 15 (4): gmr15048929 
K. Yu et al.

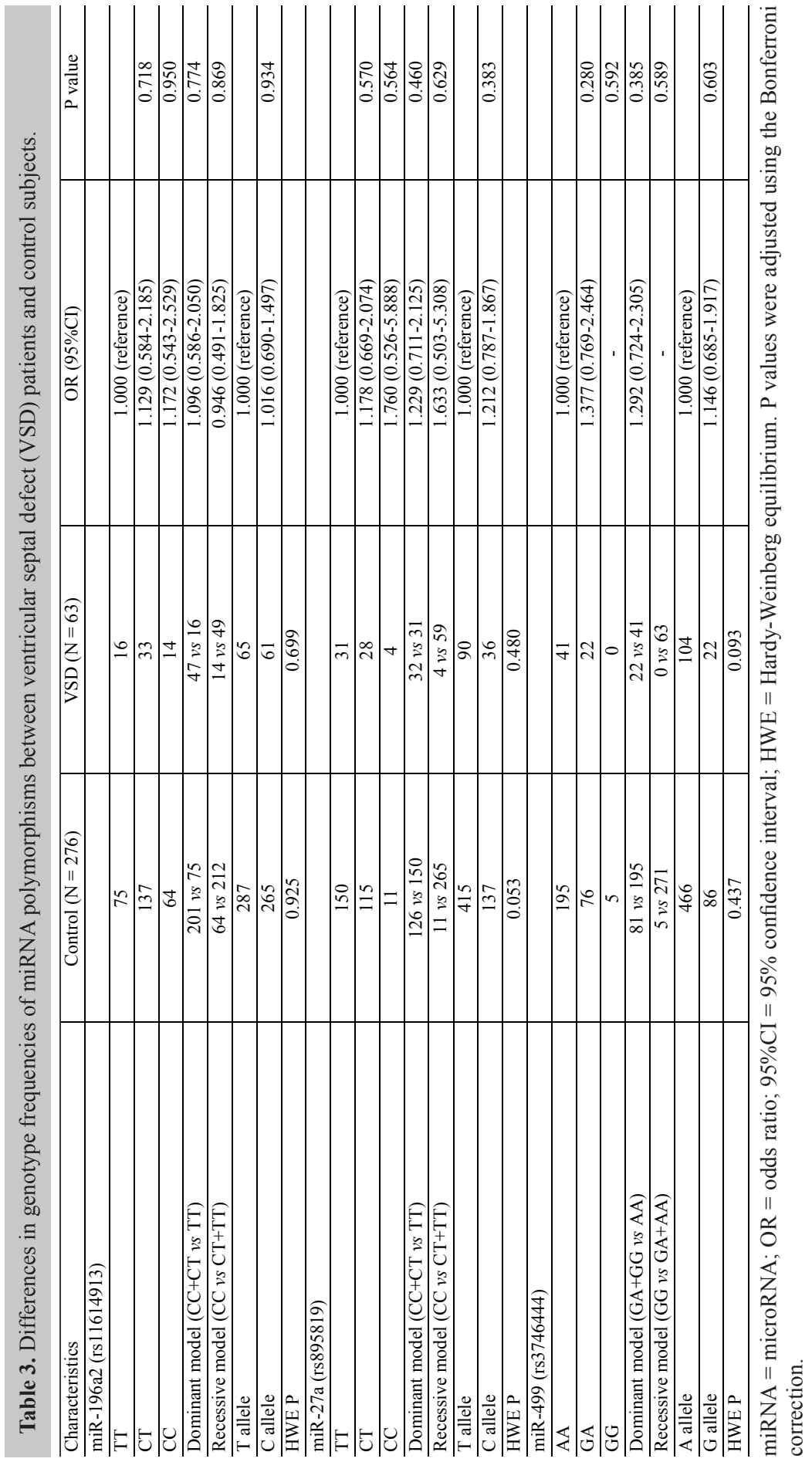

Genetics and Molecular Research 15 (4): gmr15048929 
Single nucleotide polymorphisms and congenital heart disease

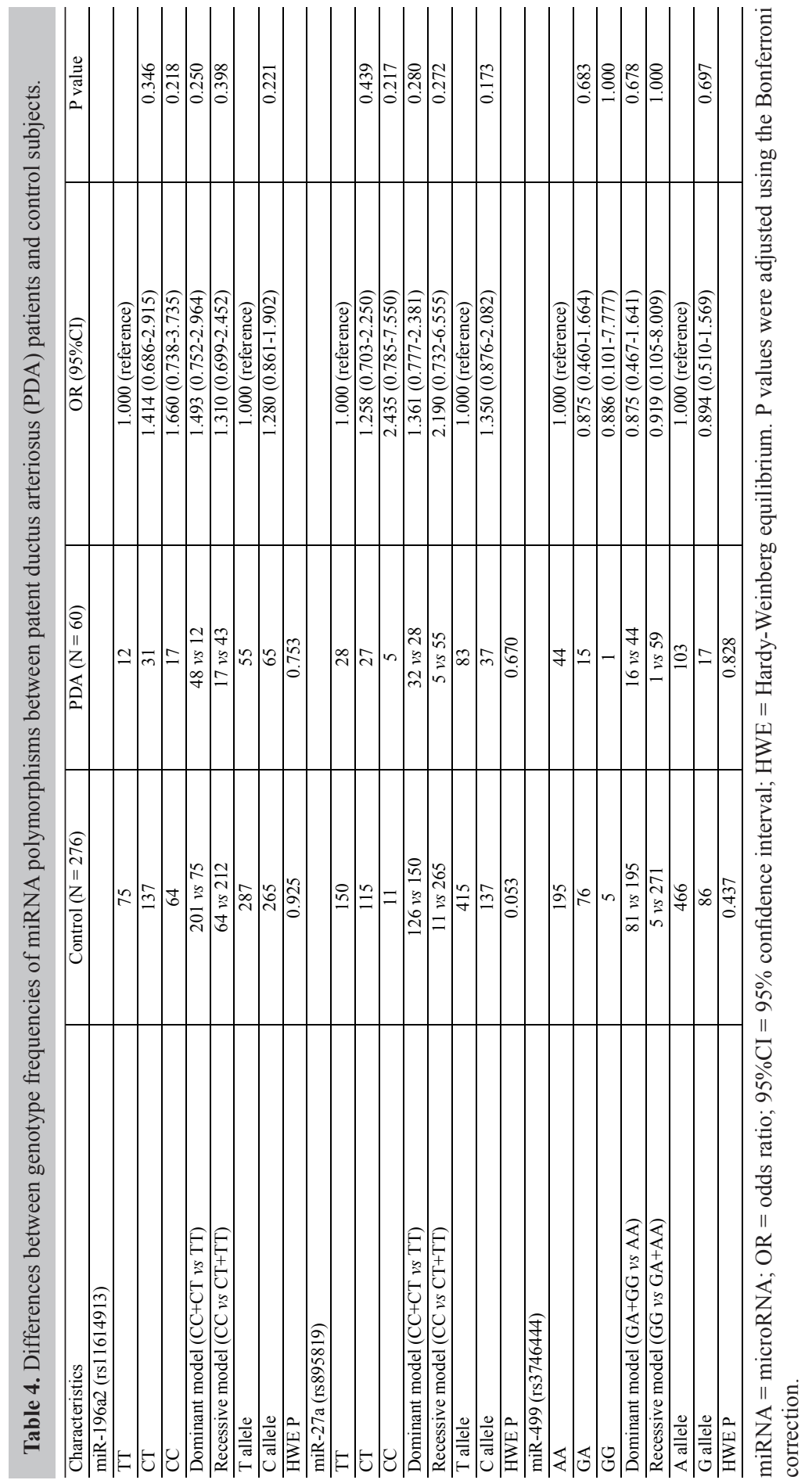

Genetics and Molecular Research 15 (4): gmr15048929 
The miR-27a (rs895819) homozygous CC variant was positively correlated with ASD $(\mathrm{OR}=1.029,95 \% \mathrm{CI}=0.400-2.645, \mathrm{P}=0.952)($ Table 2$), \mathrm{VSD}(\mathrm{OR}=1.760,95 \% \mathrm{CI}=0.526-$ $5.888, \mathrm{P}=0.564)($ Table 3$)$, and $\mathrm{PDA}(\mathrm{OR}=2.435,95 \% \mathrm{CI}=0.785-7.550, \mathrm{P}=0.217)$ (Table 4) compared with the wild-type TT variant; the $\mathrm{C}$ allele was negatively correlated with ASD $(\mathrm{OR}=0.846,95 \% \mathrm{CI}=0.615-1.165, \mathrm{P}=0.306)$ but positively correlated with VSD $(\mathrm{OR}=$ $1.760,95 \% \mathrm{CI}=0.526-5.888, \mathrm{P}=0.564)$ and $\mathrm{PDA}(\mathrm{OR}=2.435,95 \% \mathrm{CI}=0.785-7.550, \mathrm{P}=$ 0.217 ) compared with the $T$ allele (Tables $2-4$ ), although the differences were not statistically significant. Similarly, the miR-499 (rs3746444, A>G) variants GG and GA, and the G allele did not show statistically significant positive or negative correlations with ASD, VSD, or PDA compared with the wild-type AA variant and the A allele, respectively (Tables 2-4).

To confirm the accuracy of the statistically significant results, we measured their statistical power (Tables 5 and 6). In the relationship between ASD and miR-196a2 (rs11614913, $\mathrm{T}>\mathrm{C}$ ), the statistical power values were $85.90 \%$ for the CC genotype, $88.30 \%$ for the $\mathrm{C}$ allele, $76.66 \%$ for the dominant model, and $88.77 \%$ for the recessive model.

Table 5. Statistical powers of genetic associations in the present case-control study.

\begin{tabular}{l|c|c}
\hline miR-196a2 (rs11614913) & Association & Statistical power (\%) \\
\hline miR-196a2 CC & ASD & 85.90 \\
\hline miR-196a2 dominant model & ASD & 76.66 \\
\hline miR-196a2 recessive model & ASD & 88.77 \\
\hline miR-196a2 C allele & ASD & 88.30 \\
\hline
\end{tabular}

$\mathrm{ASD}=$ atrial septal defect.

Finally, we also performed an analysis by pooling the three subgroups (ASD, VSD, and PDA) into a CHD group for comparison with the control group, as shown in Table 6. The three SNPs (miR-196a2 rs11614913, miR-27a rs895819, and miR-499 rs3746444) in the control group did not show any statistically significant correlations compared with the CHD group.

Table 6. Genotype frequencies of miRNA polymorphisms between congenital heart disease (CHD) patients and control subjects.

\begin{tabular}{|c|c|c|c|c|}
\hline \multirow{2}{*}{\multicolumn{5}{|c|}{$\begin{array}{l}\text { Characteristics } \\
\text { miR-196a2 (rs11614913) }\end{array}$}} \\
\hline & & & & \\
\hline TT & 75 & 93 & 1.000 (reference) & \\
\hline CT & 137 & 152 & $0.895(0.611-1.311)$ & 0.568 \\
\hline $\mathrm{CC}$ & 64 & 52 & $0.655(0.407-1.055)$ & 0.081 \\
\hline Dominant model (CC+CT vs TT) & 201 vs 75 & 204 vs 93 & $0.818(0.570-1.175)$ & 0.277 \\
\hline Recessive model (CC vs CT+TT) & 64 vs 212 & 52 vs 245 & $0.703(0.467-1.059)$ & 0.091 \\
\hline Tallele & 287 & 338 & 1.000 (reference) & \\
\hline Callele & 265 & 256 & $0.820(0.650-1.035)$ & 0.095 \\
\hline HWE P & 0.925 & 0.454 & & \\
\hline \multicolumn{5}{|l|}{$\frac{\pi w \mathrm{~L}}{\mathrm{miR}-27 \mathrm{a}(\mathrm{r} s 895819)}$} \\
\hline TT & 150 & 165 & 1.000 (reference) & \\
\hline CT & 115 & 115 & $0.909(0.647-1.277)$ & 0.583 \\
\hline $\mathrm{CC}$ & 11 & 17 & $\frac{0.905(0.638-3.096)}{1.405)}$ & 0.397 \\
\hline Dominant model (CC+CT vs TT) & 126 vs 150 & 132 vs 165 & $0.952(0.685-1.324)$ & 0.772 \\
\hline Recessive model (CC vs CT+TT) & 11 vs 265 & 17 vs 280 & $1.463(0.673-3.181)$ & 0.335 \\
\hline $\begin{array}{ll}\text { Tallele } \\
\end{array}$ & 415 & 445 & 1.000 (reference) & \\
\hline Callele & 137 & 149 & $1.014(0.776-1.326)$ & 0.917 \\
\hline \multirow{2}{*}{\multicolumn{5}{|c|}{$\begin{array}{l}\text { HWE P } \\
\text { miR-499 (rs3746444) }\end{array}$}} \\
\hline & & & & \\
\hline AA & 195 & 209 & 1.000 (reference) & \\
\hline GA & 76 & 82 & $1.007(0.697-1.455)$ & 0.972 \\
\hline GG & 5 & 6 & $1.120(0.336-3.728)$ & 0.854 \\
\hline Dominant model $(\mathrm{GA}+\mathrm{GG} v s \mathrm{AA})$ & 81 vs 195 & 88 vs 209 & $1.014(0.708-1.452)$ & 0.944 \\
\hline Recessive model ( $\mathrm{GG} v s \mathrm{GA}+\mathrm{AA})$ & 5 vs 271 & 6 vs 291 & $1.118(0.337-3.704)$ & 0.856 \\
\hline A allele & 466 & 500 & 1.000 (reference) & \\
\hline G allele & 86 & 94 & $1.019(0.741-1.401)$ & 0.909 \\
\hline & & & & \\
\hline
\end{tabular}

miRNA $=$ microRNA; OR $=$ odds ratio; $95 \% \mathrm{CI}=95 \%$ confidence interval; HWE $=$ Hardy-Weinberg equilibrium $\mathrm{P}$ value were adjusted using the Bonferroni correction.

Genetics and Molecular Research 15 (4): gmr15048929 


\section{DISCUSSION}

SNPs located in miRNAs, so-called mirSNPs, play an important role in the development of various types of disease. Although a sizeable number of studies have been performed to investigate the role of miRNA SNPs in several types of cancer (Ryan et al., 2010), and the information obtained to date has proven that some miRNAs and their SNPs are relevant to cardiac development (Condorelli et al., 2014; Zhang et al., 2016), the relationship between miRNA SNPs and isolated CHDs, including ASD, VSD, and PDA, remains largely unknown. In the present study, we identified three efficacy outcomes from a set of four candidate miRNA SNPs. To the best of our knowledge, this is the first study to provide evidence that miR-196a2 ( $r$ 11614913 $\mathrm{T}>\mathrm{C}$ ) is a protective factor associated with ASD. The discovery of miRNA SNPs might provide a potential approach to the screening diagnosis of CHD.

mirSNPs work by regulating primary transcript levels, pre-miRNA, or pre-miRNA processing, and the interaction between miRNA and the target mRNA. They thereby influence microRNA biogenesis, the stability of mature microRNA molecules, and the efficiency of target gene regulation, as well as target specificity (Ryan et al., 2010). Given that mutation events rarely occur in certain miRNAs, functional mirSNPs as "top-level" regulators are unlikely to be tolerated and would be removed by purifying selection. Such retained mirSNPs, for example in a germline cell, can be transmitted to the next generation resulting in an altered phenotype (Mishra and Bertino, 2009), which has inspired research into the correlation between mirSNPs and disease. The importance of the mirSNP location has been debated. It has been suggested that mirSNPs, whether or not they alter the secondary structure of the miRNA precursors (Wu et al., 2008), could lead to the aberrant expression of miRNAs or target genes, suggesting that the process is more complex and subtle than predicted. Research into the underlying mechanisms has reached a bottleneck; therefore, attention has shifted to the association between mirSNPs and diseases.

During the development of the heart, Hox genes are crucial for cardiac differentiation in the drosophila larval cardiac tube, and for cardiac septation and valve formation in chicks (Chan-Thomas et al., 1993). In the genome of vertebrates, miR-196 genes are embedded within clusters of Hox transcription factor genes (Tanzer et al., 2005). The miR-196 family includes miR-196a1, miR-196a2, and miR-196b transcribed from three different genes located on chromosomes 17q21,12q13, and 7p15, respectively (Tanzer et al., 2005). HOXB8, which is essential for myeloid differentiation, might be the target of miR-196a2 (Kawasaki and Taira, 2004). In a previous study, Xu et al. (2009) found that the miR-196a2 rs11614913 CC genotype increased the risk of CHD by increasing the amount of mature miR-196a2 and affecting HOXB8 binding to mature miR-196a2, thereby impairing miR-196a-HOXB8-Sonic hedgehog (Shh) signaling. Contrary to these finding, our study showed that the miR-196a2 (rs11614913 T>C) homozygous $\mathrm{CC}$ variant and the $\mathrm{C}$ allele were negatively associated with $\mathrm{ASD}$, not VSD, PDA, or the pooled CHD group. We speculate that these conflicting results may have occurred for the following reasons. First, cases with complex cardiac abnormalities were included in $\mathrm{Xu}$ et al. (2009)'s study but excluded from our study. Second, we divided CHD into ASD, VSD, and PDA, but Xu et al. (2009) summarized a variety of CHDs as a whole object for comparison; we now know that the pathogenesis may vary in the different kinds of CHD, and statistical bias may have produced the contradictory results. Third, most controls in Xu et al. (2009)'s study had a diagnosis of trauma or infection, whereas our controls were healthy.

Even though we reviewed certain diseases such as breast cancer, there were conflicting

Genetics and Molecular Research 15 (4): gmr15048929 
results for the rs $11614913 \mathrm{CC}$ genotype and cancer risk (Wang et al., 2013). This illustrates that the mechanism of miRNAs in relation to diseases requires further exploration. Interestingly, in a study designed to confirm that the same allele (C allele) was associated with risk, the minor alleles varied (Hoffman et al., 2009). The rs11614913 SNP is a common allele in the Asian population; the minor allele is the $\mathrm{C}$ allele and its frequency is 0.449 in CHB (Han Chinese in Beijing, China) and 0.394 in JPT (Japanese in Tokyo, Japan) according to HapMap. The T allele is the minor allele in other ethnicities such as CEU (Utah residents with Northern and Western European ancestry from the CEPH collection) and YRI (Yoruba in Ibadan, Nigeria), in which the frequencies are 0.442 and 0.136 , respectively. There seems to be ethnic diversity, and the phenomena has been further confirmed by several studies (Hoffman et al., 2009). Thus, repeated investigation of the relationship between rs11614913 and ASD is required in different ethnicities.

The rs895819 SNP of miR-27a is located on chromosome 19p13.13 at position 40 relative to the first nucleotide, which is the center of the terminal loop of pre-miR-27a (Sun et al., 2010). When the loop is shortened by mutation or deletion (mirSNPs), the maturation process is harmed and miRNA secondary structure is altered, leading to reduced production of both pre-miR-27a and mature miR-27a via complex mechanisms (Zeng et al., 2005). miR27a has not been reported to be involved in CHD pathogenesis, but certain miRNAs including miR-208a, -208b, -499, and -27a have been reported to be involved in cardiac and muscle development (Callis et al., 2009). miR-27a has a stronger influence on the regulation of $\beta-\mathrm{MHC}$ expression than miR-208a, $-208 \mathrm{~b}$, or -499 . The rs3746444 SNP of miR-499, which is located in the segment of the miR-499 gene encoding the seed region of the mature passenger microRNA strand (miR-499-3p), causes a mismatch in the stem region of the miR-499 precursor. This potentially leads to a secondary structure alteration that affects the biogenesis of mature microRNA molecules, interactions with target mRNAs, and target specificity $\mathrm{Hu}$ et al., 2009). miR-499 is expressed in differentiated human cardiomyocyte progenitor cells, and together with its gene (MYH7) is strongly enriched in cardiac tissue. miR-499 plays a role in cardiomyocyte homeostasis and maintaining the differentiation state of cardiomyocytes by enhancing differentiation of human cardiomyocyte progenitor cells into cardiomyocytes (Sluijter et al., 2010). The present study did not reveal a significant correlation between miR27a (rs895819) and miR-499 (rs3746444) and ASD, VSD, or PDA. Studies with larger sample sizes are needed to confirm the relationship between these two miRNAs and CHD.

In this study, we proposed the hypothesis that life style (smoking status, alcohol consumption, weight, etc.) or its accompanying diseases (hypertension, hyperlipidemia, etc.) might not affect irreversible CHD diagnosed after birth. Therefore, this case-control study was matched only by age and gender and made no correction for multiple comparisons in case of statistical bias. Several limitations need to be addressed. First, the cases and controls were enrolled from the same hospital at the same area, so the moderate sample size may not have accurately represented the whole population. Second, although we imposed inclusion criteria on the cases and controls, maternal status before and during pregnancy (mental status, folic acid taken, working pressure, etc.), which contributes to fetal heart development, might have confounded the results. These potential risk factors require further investigation.

We determined that the miR-196a2 (rs11614913, T>C) homozygous $\mathrm{CC}$ variant and the $\mathrm{C}$ allele are associated with a significantly reduced risk of ASD. This conclusion needs to be verified by further large-scale and ethnically based cohorts. The exact role of the rs 11614913 SNP and the related molecular mechanism that occurs during ASD pathogenesis need to be elucidated.

Genetics and Molecular Research 15 (4): gmr15048929 


\section{Conflicts of interest}

The authors declare no conflict of interest.

\section{ACKNOWLEDGMENTS}

Research supported by grants from the Priority Academic Program Development of Jiangsu Higher Education Institutions (grant \#PAPD2014-2016). Dr. Wei Sun is an Assistant Fellow at the Collaborative Innovation Center for Cardiovascular Disease Translational Medicine, and Dr. Xiangqing Kong is a Fellow at the Collaborative Innovation Center for Cardiovascular Disease Translational Medicine.

\section{REFERENCES}

Bartel DP (2009). MicroRNAs: target recognition and regulatory functions. Cell 136: 215-233.http://dx.doi.org/10.1016/j. cell.2009.01.002

Bruneau BG (2008). The developmental genetics of congenital heart disease. Nature 451: 943-948. http://dx.doi. org/10.1038/nature06801

Callis TE, Pandya K, Seok HY, Tang RH, et al. (2009). MicroRNA-208a is a regulator of cardiac hypertrophy and conduction in mice. J. Clin. Invest. 119: 2772-2786. http://dx.doi.org/10.1172/JCI36154

Chan-Thomas PS, Thompson RP, Robert B, Yacoub MH, et al. (1993). Expression of homeobox genes Msx-1 (Hox-7) and Msx-2 (Hox-8) during cardiac development in the chick. Dev. Dyn. 197: 203-216. http://dx.doi.org/10.1002/ aja. 1001970305

Chen J and Wang DZ (2012). microRNAs in cardiovascular development. J. Mol. Cell. Cardiol. 52: 949-957. http://dx.doi. org/10.1016/j.yimcc.2012.01.012

Condorelli G, Latronico MV and Cavarretta E (2014). microRNAs in cardiovascular diseases: current knowledge and the road ahead. J. Am. Coll. Cardiol. 63: 2177-2187. http://dx.doi.org/10.1016/j.jacc.2014.01.050

Hoffman AE, Zheng T, Yi C, Leaderer D, et al. (2009). microRNA miR-196a-2 and breast cancer: a genetic and epigenetic association study and functional analysis. Cancer Res. 69: 5970-5977. http://dx.doi.org/10.1158/0008-5472.CAN$\underline{09-0236}$

Hoffman JI (2013). The global burden of congenital heart disease. Cardiovasc. J. Afr. 24: 141-145. http://dx.doi. org/10.5830/CVJA-2013-028

Hu Z, Liang J, Wang Z, Tian T, et al. (2009). Common genetic variants in pre-microRNAs were associated with increased risk of breast cancer in Chinese women. Hum. Mutat. 30: 79-84. http://dx.doi.org/10.1002/humu.20837

Kawasaki H and Taira K (2004). MicroRNA-196 inhibits HOXB8 expression in myeloid differentiation of HL60 cells. Nucleic Acids Symp Ser (Oxf) 2004: 211-212.http://dx.doi.org/10.1093/nass/48.1.211

Lander ES, Linton LM, Birren B, Nusbaum C, et al.; International Human Genome Sequencing Consortium (2001). Initial sequencing and analysis of the human genome. Nature 409: 860-921. http://dx.doi.org/10.1038/35057062

Lewis BP, Burge CB and Bartel DP (2005). Conserved seed pairing, often flanked by adenosines, indicates that thousands of human genes are microRNA targets. Cell 120: 15-20. http://dx.doi.org/10.1016/j.cell.2004.12.035

Li M, Li RJ, Bai H, Xiao P, et al. (2016). Association between the pre-miR-196a2 rs11614913 polymorphism and gastric cancer susceptibility in a Chinese population. Genet. Mol. Res. 15: 10.4238/gmr.15027516.

Mishra PJ and Bertino JR (2009). MicroRNA polymorphisms: the future of pharmacogenomics, molecular epidemiology and individualized medicine. Pharmacogenomics 10: 399-416. http://dx.doi.org/10.2217/14622416.10.3.399

Nishi H, Ono K, Horie T, Nagao K, et al. (2011). MicroRNA-27a regulates beta cardiac myosin heavy chain gene expression by targeting thyroid hormone receptor beta1 in neonatal rat ventricular myocytes. Mol. Cell. Biol. 31: 744-755.http://dx.doi.org/10.1128/MCB.00581-10

Patrushev LI and Kovalenko TF (2014). Functions of noncoding sequences in mammalian genomes. Biochemistry (Mosc.) 79: 1442-1469. http://dx.doi.org/10.1134/S0006297914130021

Ryan BM, Robles AI and Harris CC (2010). Genetic variation in microRNA networks: the implications for cancer research. Nat. Rev. Cancer 10: 389-402. http://dx.doi.org/10.1038/nrc2867

Saunders MA, Liang H and Li WH (2007). Human polymorphism at microRNAs and microRNA target sites. Proc. Natl. Acad. Sci. USA 104: 3300-3305. http://dx.doi.org/10.1073/pnas.0611347104

Genetics and Molecular Research 15 (4): gmr15048929 
Schonrock N, Harvey RP and Mattick JS (2012). Long noncoding RNAs in cardiac development and pathophysiology. Circ. Res. 111: 1349-1362. http://dx.doi.org/10.1161/CIRCRESAHA.112.268953

Sluijter JP, van Mil A, van Vliet P, Metz CH, et al. (2010). MicroRNA-1 and -499 regulate differentiation and proliferation in human-derived cardiomyocyte progenitor cells. Arterioscler. Thromb. Vasc. Biol. 30: 859-868. http://dx.doi. org/10.1161/ATVBAHA.109.197434

Srivastava D (2006). Making or breaking the heart: from lineage determination to morphogenesis. Cell 126: 1037-1048. http://dx.doi.org/10.1016/j.cell.2006.09.003

Sun Q, Gu H, Zeng Y, Xia Y, et al. (2010). Hsa-mir-27a genetic variant contributes to gastric cancer susceptibility through affecting miR-27a and target gene expression. Cancer Sci. 101: 2241-2247. http://dx.doi.org/10.1111/j.13497006.2010.01667.x

Tanzer A, Amemiya CT, Kim CB and Stadler PF (2005). Evolution of microRNAs located within Hox gene clusters. $J$. Exp. Zoolog. B Mol. Dev. Evol. 304: 75-85. http://dx.doi.org/10.1002/jez.b.21021

Wang N, Tian ZQ, Li Y, Zhou RM, et al. (2013). An A/G polymorphism rs3746444 in miR-499 is associated with increased cancer risk: a meta-analysis. Genet. Mol. Res. 12: 3955-3964. http://dx.doi.org/10.4238/2013.September.23.14

Wu M, Jolicoeur N, Li Z, Zhang L, et al. (2008). Genetic variations of microRNAs in human cancer and their effects on the expression of miRNAs. Carcinogenesis 29: 1710-1716. http://dx.doi.org/10.1093/carcin/bgn073

$\mathrm{Xu} \mathrm{J}, \mathrm{Hu} \mathrm{Z}, \mathrm{Xu} \mathrm{Z}, \mathrm{Gu} \mathrm{H}$, et al. (2009). Functional variant in microRNA-196a2 contributes to the susceptibility of congenital heart disease in a Chinese population. Hum. Mutat. 30: 1231-1236. http://dx.doi.org/10.1002/humu.21044

Yu ZB, Han SP, Chen X, Sun XF, et al. (2014). Systematic review of the prevalence of perinatal congenital heart disease. Chinese Journal of Evidence Based Pediatrics 9: 252-259.

Zeng Y, Yi R and Cullen BR (2005). Recognition and cleavage of primary microRNA precursors by the nuclear processing enzyme Drosha. EMBO J. 24: 138-148.http://dx.doi.org/10.1038/sj.emboj.7600491

Zhang Y, Jin SQ, Li WX, Gao GQ, et al. (2016). Association between RNF41 gene c.-206 T > A genetic polymorphism and risk of congenital heart diseases in the Chinese Mongolian population. Genet. Mol. Res. 15: 10.4238/gmr.15028089.

Genetics and Molecular Research 15 (4): gmr15048929 